\title{
ANALYSIS OF A SERIES OF INVASIVE BREAST CANCERS PERCUTANEOUS COMPLETELY RESECTED (ICPR) BY VACUUM-ASSISTED BIOPSY (VAB) OR EXCISION (VAE) AND ITS CLINICAL IMPLICATIONS
}

Henrique Lima Couto1, Carolina Nazareth Valadares¹, Aleida Nazareth Soares¹, Vivian Resende'1, Paola Hartung

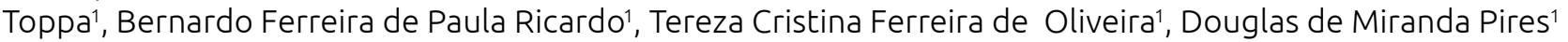

${ }^{1}$ Clínica Radiológica Ambulatorial Redimasto, Redimama Ltda - Belo Horizonte (MG), Brazil.

Objectives: To evaluate ICPR by VAB or VAE and its clinical implications. Methodology: A retrospective analysis of ICPR by VAB/VAE from January 4, 2017, to September 10, 2020, confirmed no tumor on surgical pathology. Age, imaging, pathological features, guidance approach (ultrasound $\times$ stereotaxis), and procedure $(\mathrm{VAB} \times \mathrm{VAE}$ ) were analyzed using paired $t$ test. This study was approved by Ethical Committee. Results: Twenty-one ICPR were found, patients' age ranged from 35 to 91 years (mean 61.57); 14 (66.7\%) invasive cancer (IC) (12 ductal and 2 lobular), 6 (28.6\%) IC associated with ductal carcinoma in situ (DCIS), 1 (4.7\%) IC associated with DCIS with comedonecrosis; 5 (23.8\%) nuclear grade (NG) 1, 14 (66.7\%) NG2, and 2 (9.5\%) NG3; 10 (47.6\%) histologic grade (HG) 1, 9 (42.9\%) HG2, and 2 (9.5\%) HG3; 2 (9.5\%) pN1sn (one and two nodes metastatic); 1 (4.8\%) multicentric; 3 (14.3\%) recurrences; 12 (57.1\%) Luminal A, 5 (23.8\%) Luminal B, 2 (9.5\%) Luminal Her, 1 (4.8\%) HER2 positive, and 1 (4.8\%) triple negative; 19 (90.5\%) VAEs and 2 (9.5\%) VABs; 21 (100\%) guided by ultrasound (US); 18 (85.7\%) masses, and 3 (15.3\%) masses associated with calcifications; and tumor size on image (TI) ranged from 4 to $11 \mathrm{~mm}$ (mean $7.5 \mathrm{~mm}$; SD $1.9 \mathrm{~mm}$ ) and 3 to $11 \mathrm{~mm}$ on pathological VAB/VAE specimen (TV) (mean $5.7 \mathrm{~mm}$; SD $1.9 \mathrm{~mm}$; $\mathrm{p}<0.001$ ). Conclusion: It is possible to percutaneously complete resect invasive cancers (ductal or lobular), of any IMQ subtype, smaller than $11 \mathrm{~mm}$, although 9.5\% harbors metastatic sentinel nodes. Staging (TNM) should be based on TV, although TI can be used in the absence of TV. Despite TI larger than TV, there is no clinical relevance. For prospective trials of ICPR, selecting criteria should be VAE for US masses, less than $11 \mathrm{~mm}$, of less aggressive subtypes.

Keywords: Breast Neoplasms; Biopsy; Needle; Ultrasonography; Interventional. 